\title{
Endogenous and nitrovasodilator-induced release of NO in the airways of end-stage cystic fibrosis patients
}

\section{To the Editors:}

A variety of isoforms of nitric oxide (NO) synthases are constitutively expressed in human airway and vascular endothelial cells continuously generating NO. NO plays an important role in regulating lung function in health and disease including modulation of pulmonary vascular resistance, airway calibre and host defence. Production of NO and its consumption by fluid-phase reactions can be detected and monitored in the exhaled air, providing an important window to assess the dynamics of NO metabolism in health and inflammatory lung conditions, asthma in particular [1].

A series of milestone studies uncovered a relative deficiency of pulmonary NO availability in cystic fibrosis (CF), a severe chronic inflammatory lung disease with studies generally showing reduced exhaled NO [2, 3]. However, there remains controversy regarding the anatomical localisation and mechanisms of NO deficiency, and its relationship with CF disease progression. By using extended exhaled NO analysis at different expiratory flow rates, indirect studies have attempted to distinguish NO production into bronchial and alveolar compartments based on basic models [4-7]. However, the fate of NO delivered directly to the alveoli has not been demonstrated in these patients. We aimed to investigate these issues by evaluating endogenous exhaled $\mathrm{NO}$ in end-stage $\mathrm{CF}$ in intubated patients undergoing lung transplantation and the dynamism of exhaled NO production from pulmonary microvascular metabolism of intravenous nitroglycerin (GTN) [8].

Breath-to-breath tidal measurements of $\mathrm{NO}$ concentrations in the lower airways were performed using a real-time, computercontrolled and integrated system (Logan Research Ltd 2000 and 3000 series, Rochester, UK), as described previously [8[ and in detail in the online supplementary material.

To investigate gas-phase $\mathrm{NO}$ concentrations in the lower airways of patients with end-stage $\mathrm{CF}$, seven patients (mean age, 26 yrs; five males and two females) presented for lung transplantation were studied after induction of general anaesthetic with endotracheal intubation and mechanical ventilation. CF patients were contrasted to patients undergoing routine open heart surgery for coronary artery bypass grafting (CABG; $n=4)$ exhibiting normal lung function as judged by preoperative respiratory function testing and evaluation of routine chest radiography (mean age $56 \mathrm{yrs}$ ). The studies were approved by the hospital ethics committee and patients provided written consent.

Baseline measurements to evaluate endogenous production and release of $\mathrm{NO}$ to the gas phase were performed after induction of anaesthetic, prior to excision of the lungs for transplant patients, and prior to cardiopulmonary bypass in CABG patients. This was followed in haemodynamicallystable patients with administration of a $250-\mu \mathrm{g}$ bolus of GTN into the central venous catheter with exhaled $\mathrm{NO}$ and arterial blood pressure changes recorded, as described previously [8] and in the online supplementary material.

Nearly undetectable levels of NO were found in CF patients representing an output of $7.6 \pm 6 \mathrm{ppb}$ over $30 \mathrm{~s}$. This is in contrast to patients presented for routine open heart surgery $(91.4 \pm 21 \mathrm{ppb}$ over $30 \mathrm{~s}$; fig. 1). Representative traces are shown in figure $1 \mathrm{~A}$ and $\mathrm{C}$ of the online supplementary material.

There was a significant increase in gas-phase $\mathrm{NO}$ above baseline levels by $250 \mu \mathrm{g}$ GTN boluses in CF patients $(36.7 \pm 6 \mathrm{ppb})$, which was comparable to that seen in control patients with routine open heart surgery $(48.7 \pm 4 \mathrm{ppb}$; fig. 1$)$. Representative traces of GTN-induced exhaled NO are presented in figure 1B and $\mathrm{D}$ of the online supplementary material.

These findings provide novel insights into the current debate on NO exchange dynamics in adult CF. Despite the chronic inflammatory nature of the disease, exhaled NO has been reported unchanged or low depending on severity of the disease [2, 4-7]. Our study investigating exhaled NO in ventilated patients undergoing lung transplantation clearly demonstrates a profound reduction in endogenous gas-phase $\mathrm{NO}$ in the isolated lower airways of patients with end-stage CF. This conclusion must take into consideration both methodological issues regarding tidal measurements of exhaled $\mathrm{NO}$ and the potential influence of high endogenous carbon dioxide $\left(\mathrm{CO}_{2}\right)$ on exhaled $\mathrm{NO}$. However, the same methodology was used for our control population and during the GTN experiments in the CF patients. Regarding $\mathrm{CO}_{2}$, we have not seen major effects of varying endogenous $\mathrm{CO}_{2}$ between 3-6\% (unpublished observations) but the potential effects of even higher end tidal $\mathrm{CO}_{2}$ in the $\mathrm{CF}$ patients cannot be excluded. Unpublished observations with end-stage pulmonary hypertension demonstrating better-preserved exhaled $\mathrm{NO}$ also suggest that the reduced endogenous exhaled NO in CF may be unique, and is unlikely to be the result of end-stage lung disease per se.

The exact mechanisms of relative NO deficiency in CF are not known. Recent studies utilising extended NO exchange analysis resulted in contradictory findings showing no alterations, increased or decreased bronchial fluxes and/or alveolar NO concentrations in CF children and adults [4-7]. The emerging consensus suggests that the main mechanism of NO deficiency in adult CF may relate to increased bronchial inflammation and associated defective NO synthase expression, activity and NO production or increased degradation of produced NO [2, 3, 6, 9]. The latter may involve bacterial mechanisms, catalytic and chemical consumption by fluid phase reactions, and during passage through the highly viscous mucous layer $[6,9]$.

Our study expands current understanding of alveolar NO exchange mechanisms by investigating NO fluxes arising from 

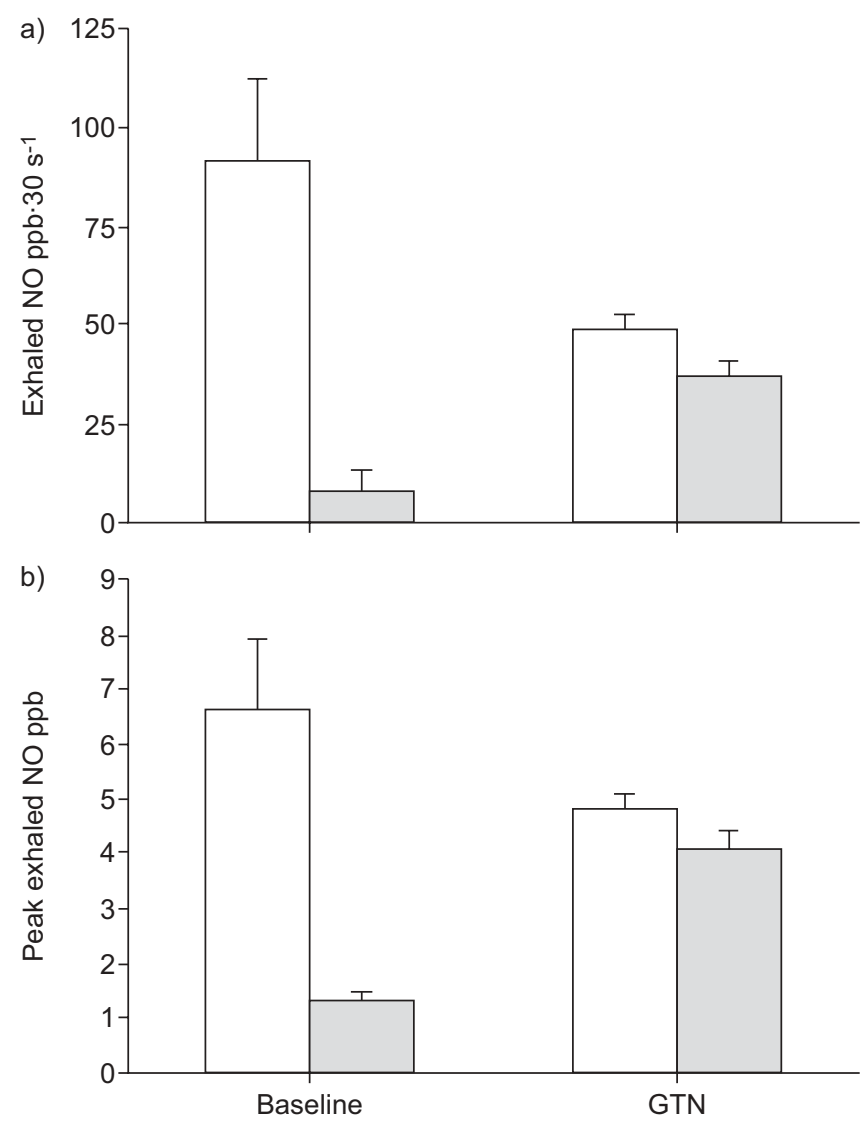

FIGURE 1. a) Area under curve over $30 \mathrm{~s}$ and b) peak exhaled nitric oxide (NO) of endogenous gas-phase NO (baseline NO) and exhaled NO in response to intravenous administration of $250-\mu \mathrm{g}$ bolus of nitroglycerin (GTN) in control patients presenting for open heart surgery for coronary arterial bypass grafting or for lung transplantation and for end-stage cystic fibrosis. GTN values represent changes over baseline.

pulmonary microvascular metabolism of exogenous nitroglycerin. Our in vivo studies in patients appear to confirm the $e x$ vivo observations of HENNO et al. [10] regarding preserved endothelium independent vasodilation by nitroprusside in isolated vascular rings of CF pulmonary arteries. Our preliminary results extend these studies to the microvasculature and airway compartments by suggesting a notably preserved ability of the end-stage CF lung microvasculature to convert GTN and release NO into the alveoli and airways without evidence of increased consumption either in the alveolar fluid or gas phase. This is remarkable in relation to both the profound reduction in endogenous NO release and the potential for pulmonary vascular dysfunction associated with end-stage $C F$ in some patients $[7,10]$. Altogether, these data lend strong support for the bronchial epithelial and mucosal consumption hypothesis for the dysregulated $\mathrm{NO}$ pathways in CF. Furthermore, they also suggest that the $\mathrm{CF}$ airways may be therapeutically targeted for exposure by $\mathrm{NO}$ through pulmonary metabolism of nitrovasodilators.

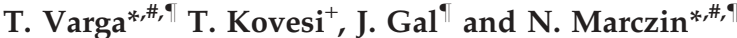

*Section of Anaesthetics, Pain Medicine and Intensive Care, Dept of Surgery and Cancer, Faculty of Medicine, Imperial College London, London, "Royal Brompton and Harefield NHS Foundation Trust, Harefield Hospital, Harefield, Middlesex, UK, "Dept of Anaesthesiology and Intensive Therapy, Faculty of Medicine, Semmelweis University, Budapest, and ${ }^{+}$Dept of Anaesthesiology and Intensive Therapy, Faculty of Medicine, Pecs University, Pecs, Hungary.

Correspondence: N. Marczin, Dept of Anaesthetics, Harefield Hospital, Harefield, Middlesex, UK, UB9 6JH. E-mail: n.marczin@ imperial.ac.uk

Statement of Interest: None declared

\section{REFERENCES}

1 Kharitonov SA, Barnes PJ. Exhaled biomarkers. Chest 2006; 130: 1541-1546.

2 Grasemann H, Michler E, Wallot M, et al. Decreased concentration of exhaled nitric oxide (NO) in patients with cystic fibrosis. Pediatr Pulmonol 1997; 24: 173-177.

3 Kelley TJ, Drumm ML. Inducible nitric oxide synthase expression is reduced in cystic fibrosis murine and human airway epithelial cells. J Clin Invest 1998; 102: 1200-1207.

4 Shin HW, Rose-Gottron CM, Sufi RS, et al. Flow-independent nitric oxide exchange parameters in cystic fibrosis. Am J Respir Crit Care Med 2002; 165: 349-357.

5 Suri R, Paraskakis E, Bush A. Alveolar, but not bronchial nitric oxide production is elevated in cystic fibrosis. Pediatr Pulmonol 2007; 42: 1215-1221.

6 Hofer M, Mueller L, Rechsteiner T, et al. Extended nitric oxide measurements in exhaled air of cystic fibrosis and healthy adults. Lung 2009; 187: 307-313.

7 Hubert D, Aubourg F, Fauroux B, et al. Exhaled nitric oxide in cystic fibrosis: relationships with airway and lung vascular impairments. Eur Respir J 2009; 34: 117-124.

8 Marczin N, Riedel B, Royston D, et al. Intravenous nitrate vasodilators and exhaled nitric oxide. Lancet 1997; 349: 1742.

9 Grasemann H, Lax H, Treseler JW, et al. Dornase alpha and exhaled NO in cystic fibrosis. Pediatr Pulmonol 2004; 38: 379-385.

10 Henno P, Maurey C, Danel C, et al. Pulmonary vascular dysfunction in end-stage cystic fibrosis: role of NF-KB and endothelin-1. Eur Respir J 2009; 34: 1329-1337.

DOI: $10.1183 / 09031936.00025610$ 